\title{
Division of labor and the evolution of task sharing in queen associations of the harvester ant Pogonomyrmex californicus
}

Received: 26 February 2003 / Revised: 13 November 2003 / Accepted: 16 December 2003 / Published online: 13 February 2004 (C) Springer-Verlag 2004

\begin{abstract}
Division of labor is a key factor in the ecological success of social groups. Recent work suggests that division of labor can emerge even without specific adaptations for task specialization and that it can appear in incipient social groups as a self-organizational property. We investigated experimentally how selection and self-organization may interact during the evolution of division of labor by examining task performance in groups of normally solitary versus normally social ant queens. We created social pairs of colony-founding queens from two populations of the ant Pogonomyrmex californicus, one in which queens are normally solitary and one in which queens form foundress groups, and observed their behavior during nest excavation. In both populations, one of the two queens usually performed most of the excavation, becoming the excavation specialist. We could predict which queen would become the specialist based on their relative propensities to perform the task in other contexts, consistent with a variancebased model of task specialization. The occurrence of specialization even when group members were not adapted to social life suggests that division of labor may well have been present in incipient queen groups. However, division of labor can result in cost skew among group members, and thus, paradoxically, within-group selection may constrain or even reduce specialization. Consistent with this effect, pairs of normally solitary queens were significantly more asymmetrical in their task
\end{abstract}

Communicated by J. Heinze

S. Helms Cahan $(\bowtie) \cdot$ J. H. Fewell

Department of Biology,

Arizona State University,

Tempe, AZ 85287-1501, USA

e-mail: Sara.HelmsCahan@ie-zea.unil.ch

Tel.: +41-21-6924193

Fax: +41-21-6924165

Present address:

S. Helms Cahan, Department of Ecology and Evolution,

Université de Lausanne,

1015 Lausanne, Switzerland performance than normally social pairs, in which both queens nearly always performed the behavior to some degree.

Keywords Division of labor - Pogonomyrmex californicus · Queen associations · Specialization · Task sharing

\section{Introduction}

Division of labor is considered a major reason for the ecological success of social groups (Wilson 1971, 1975). Although it may contribute to the maintenance of social behavior, it is unclear if division of labor, and thus any costs and benefits it confers, was an ancestral feature of social groups or is only a later adaptation. Recent models of the mechanisms underlying social organization suggest that division of labor can emerge spontaneously via selforganization and thus could be an ancestral mechanism that was present at the origin of sociality (Page 1997; Bonabeau et al. 1998; Page and Mitchell 1998; Fewell and Page 1999). In this study, we utilize naturallyoccurring intraspecific variation in sociality within an ant species to investigate whether division of labor might have occurred in incipient social groups and how it has evolved under selection.

Division of labor provides a number of important benefits for social groups. Performance of tasks by different individuals permits groups to address multiple tasks simultaneously, which can dramatically increase their ability to accomplish time-intensive tasks without sacrificing other task needs (Oster and Wilson 1978; Gamboa and Stump 1996). Specialization can also lead to more reliable and/or efficient task performance due to morphological or behavioral adaptation, learning, or the reduction in time spent in switching from one task to another (Wilson 1976; Julian and Cahan 1999). However, at the same time, division of labor may impose individuallevel costs on task specialists, who shoulder dispropor- 
tionate energetic expenditure or risk (e.g., Clutton-Brock et al. 1998; Underwood and Shapiro 1999).

Although division of labor clearly contributes to the success of extant social species, it is less clear whether the costs and benefits of division of labor played a role in the evolutionary origin of sociality. In order to have been an important factor during the initial evolution of sociality, division of labor must have been a feature of incipient social groups. Many of the models developed to explain how division of labor is generated rely on derived social adaptations, such as hormonal shifts or complex communication systems, suggesting that division of labor is a derived characteristic that evolved subsequent to sociality (reviewed by Beshers and Fewell 2001). In contrast, selforganization models of division of labor are based on simple assumptions that are likely to be met even in incipient social groups. In self-organizational models, larger-scale patterns (in this case, division of labor) emerge as a result of multiple local interactions that individually may be governed by simple behavioral rules. These processes often do not require specific social adaptations; thus, it is possible that division of labor may have been an integral part of sociality even at its origin (Helms Cahan et al. 2002). Experimentally induced associations of normally non-social individuals show pronounced task specialization for both non-reproductive and reproductive behaviors (Sakagami and Maeta 1987; Wcislo 1997; Fewell and Page 1999), consistent with the hypothesis that the basic properties of division of labor can self-organize.

Three possible self-organizational mechanisms might generate specialization in simple groups. These models share a common hypothesis that initial differences in behavioral performance generate increased divergence via self-organization. First, dominance interactions between individuals may spatially segregate dominant and subordinate individuals (Hogeweg and Hesper 1983). In the dominance model, the outcome of dominance interactions reinforces dominance relationships, so that the winner becomes more dominant and the loser less dominant (selfreinforcement). If dominance drives task performance, such as is seen in wasp systems where the queen drives foragers away from the nest (Reeve and Gamboa 1987), then division of labor results. This predicts that strong division of labor should be associated with dominance interactions between group members, and that dominance status should correlate with the type of task performed.

Division of labor can also self-organize around variance among group members in their initial propensity to perform a specific task. This model assumes that individuals perform a task when external stimuli for the task reach an internal set-point, or response threshold. Performance of the behavior by the most responsive individual lowers the stimulus level encountered by other group members, decreasing their likelihood of also performing the task (Calderone and Page 1992; Bonabeau et al. 1996; Fewell and Page 1999). Under the variance-based response threshold model, the task roles assumed by any two grouped individuals should be predictable from their relative propensities for the task in a solitary context. Finally, random variation in initial task performance could lead to specialization even among identical individuals if tasks are self-reinforcing, such that an individual is more likely to engage in tasks performed previously (Theraulaz et al. 1998). The self-reinforcement model predicts that task specialization should emerge in any group, but unlike in the variance-based model, divergence of individuals into specialist and non-specialist for a given task results from random differences in the stimulus encountered or in behavior. It further expects that differences among individuals become more cemented over successive iterations of task performance.

In this study, we investigate the mechanisms and evolution of division of labor within associations of colony-founding queens of the California harvester ant, Pogonomyrmex californicus. This species shows variation across populations in the behavior of newly-mated queens during colony founding, making it an ideal species in which to investigate the evolution of social behavior (Rissing et al. 2000). Queens in a central Arizona population exclusively found colonies solitarily (haplometrosis), as is typical for the genus (Hölldobler 1976). However, queens in a southern California population form joint starting nests (pleometrosis), containing 2-30 generally unrelated co-foundresses (Rissing et al. 2000; S. Helms Cahan, unpublished data). Pleometrotic associations most likely lead to permanent primary polygyny (personal observation), with no evidence of inter-queen aggression following worker emergence seen in some other pleometrotic species (Rissing and Pollock 1987; Bernasconi and Keller 1996). It is not currently known why populations differ in colony-founding behavior, but one selective force may be summer precipitation, which is lower and less predictable for the group-founding population. A similar trend was found in another geographically variable desert species (Helms Cahan 2001), suggesting that pleometrosis may reduce desiccation or starvation risk.

Three potentially costly tasks must be performed by queens during colony founding: nest excavation, which imposes an energetic and desiccation-resistance cost (Fewell and Page 1999; Johnson 2000); foraging, which increases predation and desiccation risk (Brown and Bonhoeffer 2003; Johnson 2002); and brood production, imposing an energetic cost (Waloff 1957). We focus here on excavation behavior, which in the group-founding population is performed primarily by a single excavation specialist, despite a reduction in survival associated with this behavioral role (Fewell and Page 1999). We investigated whether normally solitary P. californicus queens also show specialization in excavation behavior when placed into a social context. We used a series of manipulations designed to investigate whether self-organization mechanisms could explain the occurrence of division of labor within these groups. In addition to manipulations used in a previous interspecific study (Fewell and Page 1999), we also conducted detailed behavioral observations of queens to test whether dom- 
inance interactions between queens or behavioral reinforcement played a role in mediating task performance. We then compared the extent of task specialization in the solitary-founding population to that of the group-founding population to explore how division of labor has evolved under selection.

\section{Methods}

\section{Excavation behavior}

Self-organizational models predict that division of labor will emerge even in normally solitary populations in which there is no history of selection for division of labor. We investigated this by comparing division of labor in solitary-founding and group-founding populations of $P$. californicus. Solitary-founding queens were collected from the Lower Salt River Recreation Area, Maricopa Co., Arizona, on 16 May 1996 and 20 May 1999. Queens at this site do not form group nests in the field (Rissing et al. 2000). Groupfounding queens were collected from several meadows within $5 \mathrm{~km}$ of the Cameron Fire Station, San Diego Co., California, on 19-20 June 1994, 6 and 7 July 1996, and 11 July 1999. Over 90\% of queens from this area form foundress associations during nest excavation (Rissing et al. 2000; S. Helms Cahan, unpublished data). All queens were collected on the soil surface, after mating flights but before or at the beginning of nest excavation. Queens were held in individual $1.5-\mathrm{ml}$ eppendorf tubes and provided with moist paper towels during transfer to Arizona State University for experiments.

We tested the two populations separately because their mating flights are offset by several weeks and they could not be collected simultaneously. Our experimental protocol followed Fewell and Page (1999). Queens were weighed to the nearest $0.01 \mathrm{mg}$ and marked with acrylic paint on the gaster for identification. Pairs of queens of similar weight (within $0.05 \mathrm{mg}$ ), but differing in marking color, were placed into glass chambers, approximately $15 \mathrm{~cm}$ high and $5 \mathrm{~cm}$ in diameter, containing approximately $10 \mathrm{~cm}$ of moist soil collected from near the site where queens were collected. We placed heat lamps over the area where the chambers were set up and maintained a room temperature of approximately $28^{\circ} \mathrm{C}$.

Chambers were watched for one half hour out of each hour for 16-30 h. During each observation period, we scanned all chambers and recorded all observed instances of excavation behavior by each queen, defined as carrying a piece of soil in the mandibles to the soil surface. The total number of excavation trips by each queen was tallied and each queen pair was tested for equality with a $G$-test of independence. We analyzed only pairs in which at least seven trips were observed. Queens performing significantly more excavation trips in their pair were identified as High Frequency Excavators (HFE); queens performing fewer were Low Frequency Excavators (LFE). The proportion of pairs showing significant excavation differences were compared across years and populations with an $\mathrm{R} \times \mathrm{C} G$-test of Independence with post-hoc subset comparisons.

We also calculated the degree of excavation symmetry within each pair as the number of excavation trips performed by the queen performing fewer excavations divided by the number performed by the other queen. A symmetry value of 0 indicates complete asymmetry (one queen performed all excavation trips in that pair); a value of 1 indicates equal task performance. Degree of symmetry was compared across populations and years with two-way ANOVAs on arcsin-squareroot-transformed values.

If queens condition their own excavation efforts on the intensity of their partner's behavior, this should lead to a correlation between the number of excavation trips made by the two queens within a pair (Hunt and Simmons 2002). We tested for this relationship by assigning one member of each pair randomly as the focal queen, and regressing the excavation rates of non-focal queens on those of the focal queens.
Is task specialization an emergent property?

All three self-organization models propose that division of labor is an emergent property of the group; in other words, the social context generates larger differences in the task performance of group members than that predicted from their relative intrinsic propensities to perform the task (Hemelrijk 2002). To test this prediction, we created pairs of queens that had assumed the same behavioral role in the initial experiment, either two excavation specialists (HFE/HFE) or two non-specialists (LFE/LFE), to determine whether task specialization would still emerge between these queens despite roughly similar excavation histories. Only queens from significantly asymmetrical pairs from the initial experiment were used. Pairs were observed and their behavior analyzed as described above. Again, pairs were tested for excavation asymmetry with $G$-tests of independence. These manipulations, as well as the HFE/LFE pairings described below, were performed on the group-founding population in 1994, and on both populations in 1999.

\section{Dominance effects}

If task specialization were mediated by dominance interactions between queens, the extent of behavioral dominance should be associated with the level of task specialization, and dominant individuals should be significantly more (or less) likely to become the excavation specialist. We investigated this in 1996 by noting all behavioral interactions between queens during the initial pairings described above for the first full hour after they were paired. Two types of interactions were asymmetrical in nature and could be construed as dominance behaviors. The first, which we termed "standing," consisted of one queen placing her forelegs on the second queen, who lay motionless on the ground. This usually lasted 1-2 min. The second was fighting, when one queen bit the other queen on the thorax or petiole. If such interactions were observed, we classified as "dominant" the queen performing the majority of dominance behaviors. To determine the effect of dominance on task performance, we tested whether significant excavation asymmetry was associated with the occurrence of dominance interactions with a $G$-test of Independence. In addition, for all asymmetrical pairs in which a dominant could be identified, we tested for an association between dominance and excavation role with a binomial test.

\section{Role of intrinsic variability}

If task roles are mediated by intrinsic variation in excavation rates, the behavioral roles assumed by each queen of a pair should be predictable from their behavior in other contexts. We designed two manipulations to test this prediction. First, we tested whether the relative excavation rates of queens when alone could predict which queen became the excavation specialist. Queens were placed individually in soil nests, and observed as described above for 13 $14 \mathrm{~h}$. These data were collected simultaneously with the observational data on initial sets of foundress pairs. We then ranked queens within each population by the number of excavation trips, divided the set in half to create "Higher Frequency" and "Lower Frequency" subsets, and re-ranked the queens within each subset. Queens from the Higher Frequency subset were paired with queens of the same ranking from the Lower Frequency subset (i.e. the highest rate individual from subset 1 with the highest rate individual from subset 2 , etc.). We performed this manipulation on 48 of the solitary-founding and 48 of the group-founding queens collected in 1999, resulting in 20 pairs with significant differences in excavation from the solitary-founding population and 18 from the groupfounding population. Pairs were observed for $15-23 \mathrm{~h}$ as described above to determine which queen would become the excavation specialist.

If differences in task role are based on intrinsic variation, we should also be able to predict who will become the HFE in a new 
social group based on her role in previous pairs. To test this, pairs with significant excavation asymmetry were removed from experimental nests and each HFE queen was placed into a new soil-filled chamber with an unfamiliar LFE queen. Pairs were observed as described above for 19-46 h to determine whether the original HFE queen would retain her behavioral role.

Role of reinforcement

If the act of excavation increases the likelihood that an individual will excavate again, we would expect to see an increase in the level of task asymmetry over time within queen pairs. We tested this by calculating an excavation symmetry index separately for the first and second halves of the initial experiment. This was only done for data sets in which queens were observed for a total of $24 \mathrm{~h} \mathrm{(12} \mathrm{h} \mathrm{for}$ each half), in 1994 for the group-founding population and in 1996 for the solitary-founding population. Symmetry indices for the first and second halves were compared with a paired $t$-test. Note that symmetry indices for the second half could exceed 1 if the lower frequency excavator in the first half of the experiment later performed more excavations. Colonies in which no excavations were observed for either queen during one half (first or second) of the experiment were excluded from analysis.

Survival

In 1996 and 1999, nest chambers of all pairs where queens had retained their behavioral roles throughout the experiment were maintained under heat lamps for 7 weeks following excavation. We provided nests with 10-15 ml water and 10 Kentucky Bluegrass seeds weekly. After 7 weeks, we emptied all nest chambers and noted all surviving queens.

\section{Results}

Excavation behavior

\section{Frequency of task specialization}

A total of 101 pairs of queens from the solitary-founding population were observed, 59 pairs in 1996 and 42 pairs in 1999. Across both years, $70 \%$ of pairs with enough data to be analyzed were significantly asymmetrical in excavation behavior (Fig. 1). There were no significant differences between years in the proportion of asymmetrical pairs ( $G$-test of independence, year: $G_{1}=0.20$, $P>0.6$ ).

We observed 139 pairs of queens from the groupfounding population: 50 pairs in 1994, 39 pairs in 1996, and 50 pairs in 1999 (Fig. 1). Across the three years, 57\% of analyzable pairs showed significant differences in excavation behavior. The proportion also did not differ across years $\left(G_{2}=4.06, P>0.1\right)$, so interpopulation comparison was made with pooled data. When pairs that could not be analysed were excluded, the proportion of pairs that were significantly asymmetrical in the group-founding population tended to be lower than for the solitaryfounding population, with a statistical comparison very close to significance (Chi-squared test, $X^{2}{ }_{1}=3.74$, $P=0.053$ ) (Fig. 1).

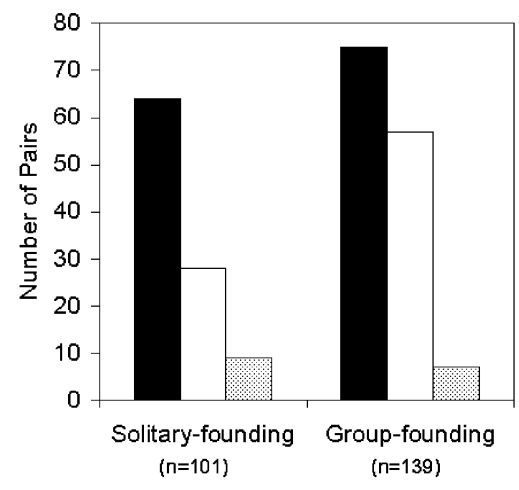

Fig. 1 The number of pairs of Pogonomyrmex californicus queens from a solitary-founding population and a group-founding population whose excavation behavior was asymmetrical (black bars), symmetrical (white bars), or too low for analysis (gray bars), pooled over all years

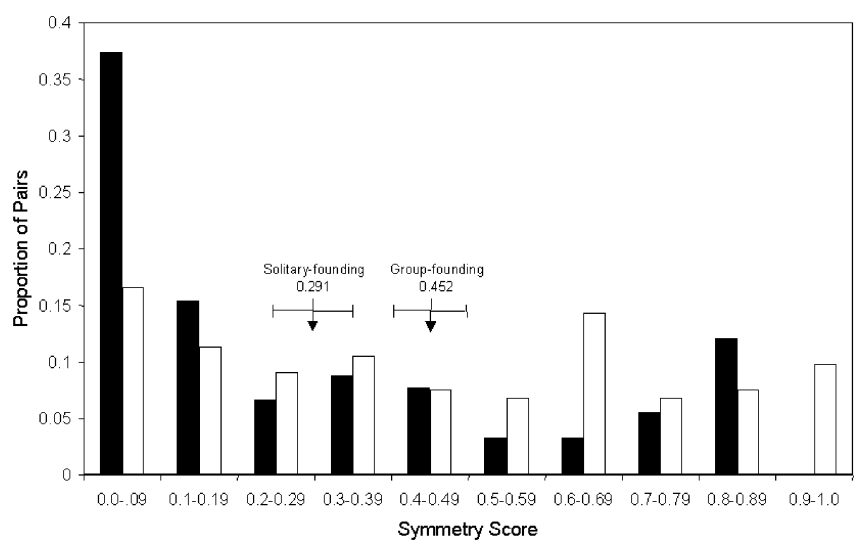

Fig. 2 Distribution of symmetry scores (LFE trips/HFE trips) for solitary-founding population (black bars) and group-founding population (white bars) of Pogonomyrmex californicus. Distributions are pooled over all years. All pairs with enough excavation trips to be analyzed were included in analysis

\section{Degree of task specialization}

The average degree of asymmetry within pairs differed between the normally solitary and normally group-founding populations (Fig. 2). Pairs of normally non-social queens were much more asymmetrical in excavation performance than social pairs (ANOVA, population: $F_{1,220}=18.65, P<0.0001$ ). A total of $24 \%$ (22 of 92) of non-social pairs showed complete asymmetry in which one of the queens performed all excavation, in contrast to $5 \%$ (7 of 132) completely asymmetrical pairs of social queens (Fig. 2). Within populations, average symmetry scores across years did not differ (year: $F_{2,219}=1.76$, $P>0.15$; year $\times$ population: $\left.F_{1,219}=0.23, P>0.60\right)$.

The relationship between excavation activities of paired queens differed across years in both populations. In the solitary-founding population, the excavation rates of the two queens were positively correlated in 1996 $\left(F_{1,57}=6.50, P=0.01\right)$ but not in $1999\left(F_{1,38}=0.11, P=0.74\right)$. 

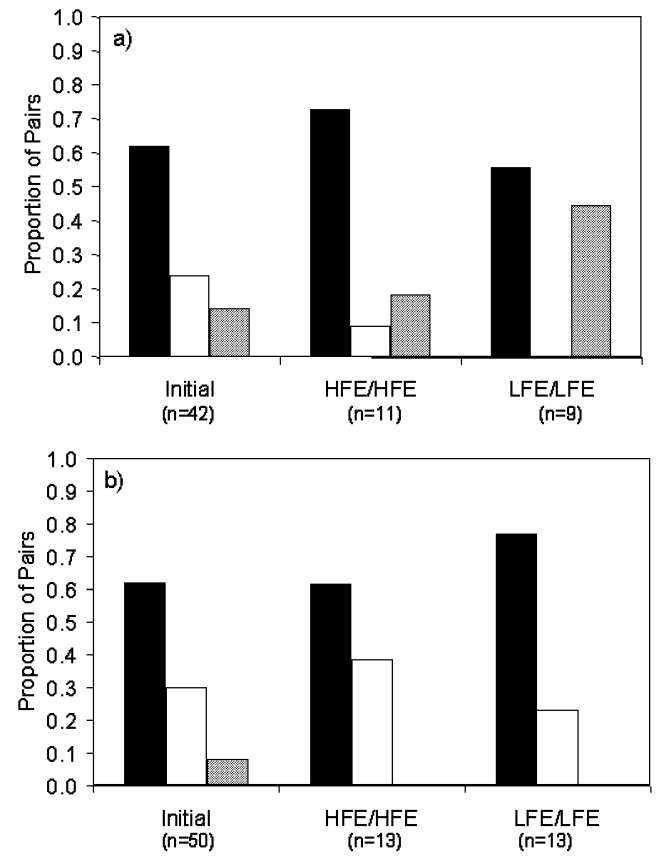

Fig. 3a, b The proportion of pairs of queens with the same original behavioral role (HFE/HFE and LFE/LFE manipulations) whose excavation behavior was asymmetrical (black bars), symmetrical (white bars), or too low for analysis (gray bars), compared with initial frequencies of excavation asymmetry. a Solitary-founding population. b Group-founding population

Excavation rates in the group-founding population were also positively correlated in $1996\left(F_{1,37}=5.47, P=0.02\right)$ but not correlated in $1994\left(F_{1,47}=0.58, P=0.45\right)$ or 1999 $\left(F_{1,47}=0.01, P=0.75\right)$.

Is task specialization an emergent property?

To test whether task specialization is generated by the social context, we created pairs of queens with similar excavation experience (either low or high) to see if task specialization would still emerge in such groups. Pairs of queens with same initial excavation role from the solitaryfounding population were just as likely to show significant task asymmetry as the original randomly assembled pairs $\left(G_{2}=0.70, P=0.71\right)$. Sample sizes are listed in Fig. 3 . Seventy-three percent of HFE/HFE pairings were asymmetrical, slightly higher than the $63 \%$ seen in original pairs from the same year. LFE/LFE pairings were significantly asymmetrical in all pairs that could be statistically analyzed. It should be noted, however, that in $44 \%$ of colonies neither queen initiated excavation, resulting in too few total excavation trips to be able to assess relative performance of the behavior (Fig. 3a).

Trends in the group-founding population were similar to the solitary-founding population. Pairs of queens of the same excavation role (HFE/HFE and LFE/LFE) were just as likely to show significant task asymmetry as the original randomly assembled pairs (HFE/HFE: $G_{1}=0.15$, $P=0.70$; LFE/LFE: $G_{1}=0.45, P=0.50$ ) (Fig. $3 b$ ).

\section{Dominance effects}

We monitored behavioral interactions between queens for the first hour in order to determine whether dominance mediates skew in excavation behavior. Initial behavioral interactions between queens from the solitary-founding population in 1996 were generally peaceful; over the first hour, one queen was observed standing on the other in five pairs, but no fighting occurred in any pair. Because so few pairs displayed any dominance, it was not possible to test for association between dominance status and task role. Fighting was observed in some pairs later on in the experiment, an average of $11.5 \pm 1.2$ (SE) h after introduction. Fights tended to occur more often in pairs that did not show significant excavation asymmetry (symmetrical pairs: 11 of 28, asymmetrical pairs: 13 of 64), but this difference was not significant $\left(G_{1}=3.49, P=0.06\right)$. When fighting occurred in asymmetrical pairs, task role was associated with individual aggressive behavior. Ten fights in pairs showing significant excavation asymmetry were clearly initiated by one queen; in nine cases, the aggressor was the HFE queen, significantly more than expected by chance (Binomial test, $P=0.05$ ). Queens engaged in nest construction were also observed removing the second queen from the nest entrance. In $21 \%$ of pairs (19 of 92), one queen was observed repeatedly dragging or carrying the other queen from within the nest hole and placing her on the soil surface. Dragging behavior occurred an average of $7.1 \pm 1.1 \mathrm{~h}$ after pairs were introduced. The removed queen was generally immobile for 5-10 s and then usually re-entered the nest hole. Dragging behavior was also not associated with whether a pair was asymmetrical in excavation behavior $\left(G_{1}=0.73, P=0.39\right)$; when dragging occurred in significantly asymmetrical pairs, the queen performing the behavior often was the HFE queen, although this pattern was not significant (6 of 8 ; Binomial test, $P>0.1$ ). In all cases, both queens were found in the nest hole at the end of the experimental observations.

Queens from the group-founding population in 1996 showed no initial fighting in the first hour, but standing behavior was observed in 13 of the 38 pairs, significantly more often than that observed for the solitary-founding population ( $G$-test of independence, $G_{1}=16.89$, $P<0.0001)$. In all cases only one of the two queens displayed the behavior. Standing was not associated with whether the pair showed subsequent excavation differences (symmetrical pairs: 4 of 16, asymmetrical pairs: 9 of $\left.22 ; G_{1}=1.06, P=0.30\right)$. In pairs displaying excavation differences, the dominant queen was not significantly more likely to take on a particular behavioral role (Binomial test, $P>0.10$ ).

Interactions between queens from the group-founding population tended to remain peaceful throughout excavation. Only one fight was observed in 1996, significantly 
fewer than for the solitary-founding population $\left(G_{1}=\right.$ 12.43, $P<0.0005)$, and no queens displayed dragging behavior, again significantly less than for the solitaryfounding population $\left(G_{1}=11.16, P<0.0005\right)$. Indeed, only two fights were observed in all three years of observation, while only a single queen displayed dragging behavior. As with the solitary-founding population, in all cases both queens cohabited the same nest hole at the end of the experiment.

\section{Role of intrinsic variability}

\section{Can excavation when alone predict task role?}

If task specialization is driven by intrinsic variance in excavation propensity, the relative propensities of group members should determine which one becomes the excavation specialist. We tested this by assessing excavation rates for queens kept alone and then placing them in pairs to determine if the queen with the higher initial rate would become the specialist. In both populations, individuals with higher excavation rates when alone tended to become the HFE when placed in pairs. The higher frequency excavator became the excavation specialist in 11 of 16 analyzed pairs of queens from the solitary-founding population. Four additional pairs could not be used because identifying paint marks were lost or obscured. In the group-founding population, the higher frequency queen became the excavation specialist in 11 of 16 pairs. Two additional pairs could not be analyzed because neither queen initiated excavation behavior. Although neither was significantly different from random when considered separately, relative excavation rate did significantly predict task role when data from both populations were pooled $\left(X^{2}{ }_{1}=4.0, P<0.05\right)$.

Data on the excavation rates of single queens were collected simultaneously with those for initial queen pairs (before manipulations); this allowed us to compare average excavation rates of queens when alone versus in groups. Single queens from the solitary-founding population initiated excavation behavior as quickly as HFE queens and significantly earlier than LFE queens from pairs (ANOVA, $F_{2,68}=9.28, P<0.001$; Bonferroni pairwise comparisons: singles vs HFE, $P=0.13$; singles vs LFE, $P<0.001)$. Total excavation rate of single queens was significantly higher than either HFE or LFE queens $\left(F_{2,68}=13.30, P<0.001\right.$; singles vs HFE, $P<0.001$; singles vs LFE, $P=0.002$ ).

In the group-founding population, single queens initiated excavation significantly later than HFE queens and were not significantly different from LFE queens from pairs $\left(F_{2,87}=3.18, P<0.05\right.$; singles vs HFE, $P=0.04$; singles vs LFE, $P=1.00$ ). Excavation rates of single queens were significantly lower than that of HFE but higher than that of LFE queens $\left(F_{2,87}=65.737, P<0.001\right.$; singles vs HFE, $P<0.001$; singles vs LFE, $P<0.001)$.

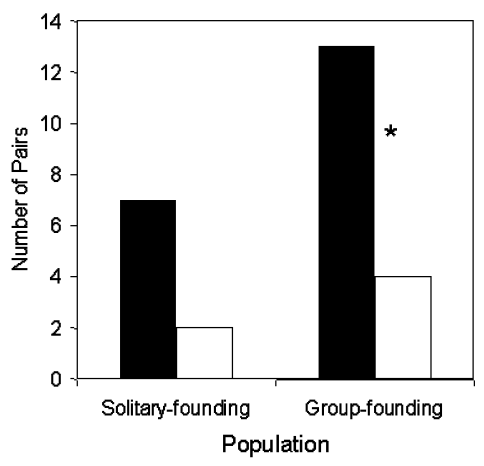

Fig. 4 Number of pairs in which queens retained their excavation roles (black bars) or switched excavation roles (white bars) when paired with an unfamiliar queen of a different previous task role (HFE/LFE manipulation). Asterisk indicates that initial roles significantly predicted task roles in second pairs (Binomial test, $P<0.05)$

\section{Can excavation in previous pairs predict task role?}

The variance model also predicts that HFE queens should have a higher intrinsic propensity to dig than LFE queens, and thus these two classes should always retain these roles when placed together in groups. HFE queens from the solitary-founding population often retained their task role when placed with an unfamiliar LFE queen, although this pattern was not statistically significant $\left(X^{2}=2.78, P=0.10\right)$ (Fig. 4). HFE queens from the group-founding population also retained their HFE role when paired with an unfamiliar LFE queen in 13 of 17 cases, significantly more often than expected by chance $\left(X^{2}{ }_{1}=4.76, P=0.03\right)$ (Fig. 4).

\section{Role of reinforcement}

If reinforcement were driving task specialization, we would predict to see in an increase in task specialization throughout the course of nest construction. This was not observed; in fact, in both populations queens became significantly more symmetrical in excavation during the second $12 \mathrm{~h}$ of observation (paired $t$-tests of symmetry indexes, solitary-founding population: $0.25 \pm 0.04$ to $0.50 \pm 0.10, t_{49}=-2.37, P<0.05$; group-founding population: $0.19 \pm 0.04$ to $\left.0.43 \pm 0.07, t_{41}=-3.23, P<0.005\right)$.

\section{Relative Survival}

In general, queens from the same pair often were either both alive or both dead when chambers were emptied after 7 weeks. In some chambers, however, only a single queen remained alive. This tended to be the LFE queen in both populations (Solitary-founding population: 12 of 18 pairs; group-founding population: 9 of 13 pairs; $68 \%$ across both populations pooled), although this difference 
was not statistically significant when considered either separately or pooled.

\section{Discussion}

Our results show that division of labor can emerge within associations of normally solitary queens. Pairs of queens from a completely solitary-founding population showed asymmetrical excavation behavior when placed into a social context at a frequency indistinguishable from those from a normally social population. In fact, levels of asymmetry within the pairs of normally solitary queens were actually higher than those of the group-founding queens. Given that other species within the genus are uniformly haplometrotic (Hölldobler 1976; Johnson 1998), it is most likely that solitary colony founding is the ancestral state in P. californicus. Thus, the occurrence of task specialization appears to be an inherent feature of forming a group, rather than requiring a secondary adaptation subsequent to the evolution of sociality.

The pattern of task specialization seen in queen pairs is consistent with the idea that self-organization processes can produce division of labor as an emergent property of the group. The statistical difference in excavation rates between queens was not merely due to pairing individuals that intrinsically differed in their propensities for excavation. Pairs of individuals who originally took on the same behavioral role, and who should have had roughly similar propensities to excavate, showed task asymmetry as frequently as random pairings. Such enhancement of task asymmetry indicates that individuals are responding to the social context itself and altering their excavation behavior when in a group. Indeed, queens that assumed the LFE role showed far lower excavation behavior than they did either when alone or when they assumed the HFE role. Similar emergence of task roles has been shown for Pogonomyrmex barbatus, another species that founds colonies solitarily (Fewell and Page 1999).

The fact that this process was successful in producing task specialization between normally solitary queens as extreme as that shown by normally social queens in all of our experimental manipulations suggests that the automatic division of labor produced by self-organization may be sufficient to explain behavioral specialization within the social population as well. The foundress association model is a good comparative fit for small communal groups found in many social taxa, because ant foundresses are generally unrelated (Hagen et al. 1988). It is difficult to assess whether this mechanism is likely to underlie division of labor in more complex societies. However, the basic assumptions of the model are consistent with work on task allocation in honeybee workers, suggesting that variance in thresholds for task stimuli can underlie the occurrence of distinct task specialists in a variety of different tasks, including nectar and pollen foraging (Robinson and Page 1989a, 1989b; Robinson 1992; Fewell and Page 1993, 2000; Page and Mitchell 1998). Such a mechanism is also likely important in regulating division of labor across multiple tasks, as the effect of performance of one task (e.g., nest repair) can lower the stimulus for that task but increase stimuli for others (foraging: Gordon 1989; Jeanne 1996).

Overall, our experiments suggest that intrinsic variance in excavation propensities, rather than dominance interactions or self-reinforcement, is the mechanism underlying the emergence of task specialization. Dominance behavior was not associated with the extent of task specialization, suggesting that dominance-based spatial segregation was not responsible for task differences. Normally solitary queens showed little initial aggression; when aggression did occur, it emerged well after excavation was initiated and was not significantly associated with the degree of task specialization. Likewise, the degree of asymmetry in excavation did not increase over time, suggesting that reinforcement is unlikely to be the primary mediator of excavation specialization. However, individual behavior when solitary and in previous pairs did predict who would become the task specialist, as expected from a variance-based model (Figs. 3, 4). Such evidence does not exclude a role for self-reinforcement, as individual excavation propensity was ascertained by allowing queens to excavate, and thus queens with different initial rates also varied in prior task performance (and therefore the potential for self-reinforcement) when placed in pairs. However, only the variance-based model predicts strong task specialization between queens if both have had extensive prior excavation experience, because in this case excavation should already be fully reinforced in both queens while intrinsic propensities would still show some variation. We created such groups by pairing two unfamiliar HFE queens; such pairs showed even higher frequencies of task asymmetry than randomly assembled pairs, with one queen from each pair substantially reducing her previous excavation rate (Fig. 3).

In addition to helping to understand the origins of division of labor, comparing levels of task specialization between the normally solitary and normally social populations also allows us to explore how division of labor has evolved under selection within social groups. The overall frequency of task specialization was somewhat lower in the social population (Fig. 1), and pairs of queens from the group-founding population consistently showed lower levels of task asymmetry than their solitaryfounding population counterparts (Fig. 2). The difference suggests that the evolutionary trajectory of the social population has been a progressive reduction in excavation specialization, as might be expected if excavation were an individually costly task. Excavation does appear to be costly, especially for desert ants such as P. californicus, as abrasion of the cuticle has been shown to double the rate of cuticular water loss (Johnson 2000; Helms Cahan, unpublished data). Even though this study was conducted in a benign laboratory environment, HFE queens tended to suffer higher mortality while the first workers were being raised. Even stronger evidence of a survival cost to excavation was found in P. barbatus (Fewell and Page 1999). If the individual-level costs of specialization are 
high, yet task specialization is automatically generated by group formation, more highly motivated queens may be selected to defect from the group rather than take on the altruistic specialist role. This may explain why group colony founding has not evolved in P. barbatus despite high density and competition between starting colonies, characteristics common to group-founding species (Bernasconi and Strassmann 1999).

Interestingly, excavation asymmetry is common despite such costs even in the normally group-founding population of $P$. californicus. There are two possible reasons why such asymmetry is maintained. First, division of labor may have strong colony-level benefits that outweigh the individual benefits of reduced specialization, such as increased excavation efficiency, earlier egglaying, or increased anti-predator vigilance during nest excavation. Alternatively, selection against specialization may have occurred, but as task performance began to be equalized, the relative costs of specialists and nonspecialists became more similar and thus weakened selection for additional changes in performance. The observed extent of sharing may redistribute costs enough to make it individually beneficial for the specialist to perform the task to make sure the nest is constructed regardless of whether or not the second queen makes more than a minimal contribution to excavation. This may be especially true if the main cost is cuticular abrasion, which most likely has a saturating rather than a linear cost function. If the cost per unit effort decreases with total effort, queens could have closely matched cost levels despite strong differences in performance of the task.

How has reduction in task asymmetry been achieved? It should not be in the interest of a non-specialist to increase her work level, yet social LFE queens appeared more willing to perform at least some excavation behavior than their non-social counterparts. This difference was not due to increased dominance interactions or physical damage to LFE queens in the solitary-founding population, as such interactions actually were more common in pairs of group-founding queens. Moreover, when dominance interactions did occur, they were unrelated to whether pairs showed task asymmetry. Such a result may be expected if each queen actively modulates her own effort based on the effort expended by her co-foundress (Trivers 1971; Reeve and Keller 1997). This hypothesis predicts that the overall excavation rates of co-foundresses should be positively correlated in the group-founding population. We found no clear evidence that such a correlation occurs. A positive relationship was found only in one year, in both the solitary-founding and groupfounding populations, suggesting that conditional investment cannot explain systematic differences between populations in degree of excavation asymmetry.

Instead, we propose that increased excavation by LFE queens is an indirect result of selection on grouping behavior. Comparison of relative task performance by queens of the two populations when kept alone suggests that the presence of a conspecific queen alters internal motivation to perform excavation behavior differently in the two groups, inhibiting excavation in the solitaryfounding population but stimulating excavation in the group-founding population. Queens from the solitaryfounding population kept alone showed a high rate of excavation behavior, significantly higher than both HFE and LFE queens from the same year. In contrast, groupfounding queens were reluctant to excavate alone, with an increased latency in initiation of the behavior and an overall excavation rate intermediate between HFE and LFE values. Queens of other group-founding species have also been shown to place a higher priority on finding cofoundresses than on locating an appropriate nest site or initiating excavation (Bartz and Hölldobler 1982; Krebs and Rissing 1991; Sommer and Hölldobler 1995; Cahan et al. 1998). This suggests that social queens use the presence of conspecifics as an additional cue to begin excavation that is likely independent of the direct stimuli provided by the soil substrate and thus not as subject to inhibition by the behavior of the task specialist.

In summary, while division of labor appears to be an automatic property of social groups, selection for grouping behavior can reduce the extent of its expression in queen associations. Division of excavation behavior appears to be influenced by two opposing mechanisms, resulting in common, but less pronounced, division of labor. The social context enhances intrinsic variability in task performance among individuals, generating asymmetries in task performance even when intrinsic variance between group members is relatively low. At the same time, however, selection for individuals preferentially joining groups rather than founding a colony alone can lead to facilitation of excavation behavior by social contact, such that all individuals are more likely to perform excavation when in groups despite the tendency to divide such behavior based on the level of task stimuli alone. Social facilitation results in more task sharing, and therefore more sharing of costs associated with the task, among co-founding queens. This reduces the individuallevel costs of sociality for task specialists and could reinforce selection for grouping behavior.

Acknowledgments Our heartfelt thanks to the many people from the Social Insect Research Group at ASU who contributed to watching queens at all hours, especially Glennis Julian and Ekwutosi Okoroh. Thanks also to Bob Johnson and Liz Smith for queen collection in 1994, and to Emma Harrison for collection and weighing assistance. This work was supported in part by an NIMH grant R29 MH51329 to J.H.F. Additional financial support during manuscript preparation was provided by grants from the Swiss National Foundation to L. Keller.

\section{References}

Bartz SH, Hölldobler B (1982) Colony founding in Myrmecocystus mimicus (Hymenoptera: Formicidae) and the evolution of foundress associations. Behav Ecol Sociobiol 10:137-147

Bernasconi G, Keller L (1996) Reproductive conflicts in cooperative associations of fire ant queens. Proc R Soc Lond B 263:509-515 
Bernasconi G, Strassmann JE (1999) Cooperation among unrelated individuals: the ant foundress case. Trends Ecol Evol 14: 477482

Beshers SN, Fewell JH (2001) Models of division of labor in social insects. Annu Rev Entomol 46:413-440

Bonabeau E, Theraulaz G, Deneubourg J-L (1996) Quantitative study of the fixed threshold model for the regulation of division of labour in insect societies. Proc R Soc Lond B 263:15651569

Bonabeau E, Theraulaz G, Deneubourg J-L (1998) Fixed response thresholds and the regulation of division of labor in insect societies. Bull Math Biol 60:753-807

Brown MJF, Bonhoeffer S (2003) On the evolution of claustral colony founding in ants. Evol Ecol Res 5:305-313

Cahan S, Helms KR, Rissing SW (1998) An abrupt transition in colony founding behaviour in the ant Messor pergandei. Anim Behav 55:1583-1594

Calderone NW, Page RE (1992) Effects of interactions among genotypically diverse nestmates on task specialization by foraging honey-bees. Behav Ecol Sociobiol 30:219-226

Clutton-Brock TH, Gaynor D, Kansky R, MacColl ADC, McIlrath G, Chadwick P, Brotherton PNM, O'Riain JM, Manser M, Skinner JD (1998) Costs of cooperative behaviour in suricates (Suricata suricatta). Proc R Soc Lond B 265:185-190

Fewell JH, Page RE (1993) Genotypic variation in foraging responses to environmental stimuli by honey-bees, Apis mellifera. Experientia 49:1106-1112

Fewell JH, Page RE (1999) The emergence of division of labour in forced associations of normally solitary ant queens. Evol Ecol Res 1:537-548

Fewell JH, Page RE (2000) Colony-level selection effects on individual and colony foraging task performance in honeybees, Apis mellifera L. Behav Ecol Sociobiol 48:173-181

Gamboa GJ, Stump KA (1996) The timing of conflict and cooperation among cofoundresses of the social wasp Polistes fuscatus. Can J Zool 74:70-74

Gordon DM (1989) Dynamics of tasks switching in harvester ant. Anim Behav 38:194-204

Hagen RH, Smith DR, Rissing SW (1988) Genetic relatedness among co-foundresses of two desert ants Veromessor pergandei and Acromyrmex versicolor. Psyche 95:191-201

Helms Cahan S (2001) Ecological variation across a behavioral transition zone in the ant Messor pergandei. Oecologia 129:629-635

Helms Cahan S, Blumstein DT, Sundström L, Liebig J, Griffin A (2002) Social trajectories and the evolution of social behavior. Oikos 96:206-216

Hemelrijk CK (2002) Understanding social behaviour with the help of complexity science. Ethology 108:655-671

Hogeweg P, Hesper B (1983) The ontogeny of interaction structure in bumble bee colonies: a MIRROR model. Behav Ecol Sociobiol 12:271-283

Hölldobler B (1976) Recruitment behavior, home range orientation and territoriality in harvester ants, Pogonomyrmex. Behav Ecol Sociobiol 1:3-44

Hunt J, Simmons LW (2002) Behavioural dynamics of biparental care in the dung beetle Onthophagus taurus. Anim Behav 64:65-75

Jeanne RL (1996) Regulation of nest construction behaviour in Polybia occidentalis. Anim Behav 52:473-488

Johnson RA (1998) Foundress survival and brood production in the desert seed-harvester ants Pogonomyrmex rugosus and $P$. barbatus. Insectes Soc 45:255-266

Johnson RA (2000) Water loss in desert ants: caste variation and the effect of cuticle abrasion. Physiol Entomol 25:48-53
Johnson RA (2002) Semi-claustral colony founding in the seedharvester ant Pogonomyrmex californicus: a comparative analysis of colony founding strategies. Oecologia 132:60-67

Julian GE, Cahan S (1999) Undertaking specialization in the desert leaf-cutter ant, Acromyrmex versicolor. Anim Behav 58:437442

Krebs RA, Rissing SW (1991) Preference for larger foundress associations in the desert ant Messor pergandei. Anim Behav 41:361-363

Oster GF, Wilson EO (1978) Caste and ecology in social insects. Princeton University Press, Princeton, N.J.

Page RE (1997) The evolution of insect societies. Endeavor $21: 114-120$

Page RE, Mitchell SD (1998) Self-organization and the evolution of division of labor. Apidologie 29:171-190

Reeve HK, Gamboa GJ (1987) Queen regulation of worker foraging in paper wasps : a social feedback control system. Behaviour 102:147-167

Reeve HK, Keller L (1997) Reproductive bribing and policing evolutionary mechanisms for the suppression of within-group selfishness. Am Nat 150: S42-S58

Rissing SW, Pollock GB (1987) Queen aggression, pleometrotic advantage and brood raiding in the ant Veromessor pergandei. Anim Behav 35:975-981

Rissing SW, Johnson RA, Martin JW (2000) Colony founding behavior of some desert ants: geographic variation in metrosis. Psyche 103:95-101

Robinson GE (1992) Regulation of division of labor in insect societies. Annu Rev Entomol 37:637-665

Robinson GE, Page REJ (1989a) Genetic basis for division of labor in an insect society. In: Breed MD, Page REJ (eds) The genetics of social evolution. Westview, Boulder, Colo., pp 61-80

Robinson GE, Page RE (1989b). Genetic determination of nectar foraging, pollen foraging, and nest-site scouting in honey bee colonies. Behav Ecol Sociobiol 24:317-323

Sakagami SF, Maeta Y (1987) Sociality, induced and/or natural, in the basically solitary small carpenter bees. In: Ito Y, Brown JL, Kikkawa J (eds) Animal societies: theories and facts. Japan Science Society, Tokyo, pp. 1-16

Sommer K, Hölldobler B (1995) Colony founding by queen association and determinants of reduction in queen number in the ant Lasius niger. Anim Behav 50:287-294

Theraulaz G, Bonabeau E, Deneubourg JL (1998) Response threshold reinforcement and division of labour in insect societies. Proc R Soc Lond B 265:327-332

Trivers RL (1971) Evolution of reciprocal altruism. Quart Rev Biol 46:35-57

Underwood DLA, Shapiro AM (1999) Evidence for division of labor in the social caterpillar Eucheira socialis. Behav Ecol Sociobiol 46:228-236

Waloff N (1957) The effect of the number of queens of the ant Lasius flavus on their survival and on the rate of development of the first brood. Insectes Soc 4:391-408

Wcislo WT (1997) Social interaction and behavioral context in a largely solitary bee, Lasioglossum figueresi. Insectes Soc 44:199-208

Wilson EO (1971) The insect societies. Harvard University Press, Cambridge, Mass.

Wilson EO (1975) Sociobiology. Harvard University Press, Cambridge, Mass.

Wilson EO (1976) Behavioral discretization and the number of castes in an ant species. Behav Ecol Sociobiol 1:141-154 